\title{
A Supervisory Control-Based Navigation Architecture for Multiple Autonomous Robots in Industry 4.0 Environments *
}

\author{
Antonio G. C. Gonzalez* Marcos V. S. Alves* \\ Lilian K. Carvalho* João C. Basilio* \\ * COPPE Programa de Engenharia Elétrica, Universidade Federal do \\ Rio de Janeiro, RJ, (e-mails: angacego@poli.ufrj.br; \\ mvalves@poli.ufrj.br; lilian@dee.ufrj.br; basilio@dee.ufrj.br).
}

\begin{abstract}
The industry 4.0 paradigm is usually associated with large factories with several smart machines that demand a powerful and versatile connectivity infrastructure. In this context, a single robot is seldom able to deal with the logistics of an entire factory, being necessary the use of multiple robots. Thus, in this paper we extend the DES-based navigation architecture for a single robot, previously proposed in Gonzalez et al. (2018), to deal with multiple mobile robots.
\end{abstract}

Keywords: Discrete Event Systems, Mobile Robots, Industry 4.0, Supervisory Control.

\section{INTRODUCTION}

In recent years, a new concept of industry, called Industry 4.0 (Gilchrist, 2016), has emerged and is currently adopted to denominate the trends of automation and data exchange in manufacturing technologies, in the context of "smart factories" (Wang et al., 2016). Some of the main fundamentals of Industry 4.0 are cyber-physical systems (Jirkovsky et al., 2017), internet of things (Da Xu et al., 2014), and mobile robots (Kehoe et al., 2015).

The Discrete Event System (DES) formalism has been proved suitable to deal with mobile robot navigation. In Roszkowska (2002), a DES-based supervisory controller that ensures collision and deadlock avoidance for a group of robots is proposed. In Košecká and Bajcsy (1994), automaton-based models for mobile robot navigation were used to show the viability of using DES theory in the modeling, analysis, and synthesis of behaviors applied to the navigation of a mobile robot. Structures for representing the environment and some specifications were presented in Iqbal et al. (2012). In Fabre and Jezequel (2009), the path planning problem to decentralized systems with action costs was addressed by using several weighted automata. In Kloetzer and Mahulea (2016), a formal method based on Linear Temporal Logic (LTL) has been employed to describe and model specifications in mobile robot navigation. A formal synthesis of supervisory control software for multiple robot systems was developed in Hill and Lafortune (2017). In Gonzalez et al. (2018) a DES-based navigation architecture for a single-robot in Industry 4.0 environments was introduced. In Tatsumoto et al. (2018) an online supervisory control approach based on limited lookahead policy for multiple robots navigation

\footnotetext{
* This work has been supported in part by the Coordenação de Aperfeiçoamento de Pessoal de Nível Superior (CAPES), Finance Code 001, and the Brazilian Research Council (CNPq), grant number $309.652 / 2017-0$.
}

was presented. In Dulce-Galindo et al. (2019) and in Basile et al. (2019), multiple robots navigation systems were proposed, with a clear focus on task assignment algorithms. The approach proposed in Dulce-Galindo et al. (2019) is centralized and automaton-based, while the one proposed in Basile et al. (2019) is decentralized (auction-based) and colored modified hybrid petri net-based.

The industry 4.0 paradigm is usually associated with large factories with several smart machines that demand a powerful and versatile connectivity infrastructure. Thus, in this context, a single robot is seldom able to deal with the logistics of a entire factory, being necessary the use of multiple robots. In order to circumventing the singlerobot limitation of the navigation architecture proposed in Gonzalez et al. (2018), in this paper, we extend the DESbased navigation proposed there to deal with multiple mobile robots.

\section{BACKGROUND PRELIMINARIES}

Let $G=\left(X, \Sigma, f, \Gamma, x_{0}, X_{m}\right)$ denote a deterministic finite automaton that models a Discrete Event System (DES), where $X$ is the finite set of states, $\Sigma$ is the finite set of events, $f: X \times \Sigma \rightarrow X$ is the transition function, partially defined over its domain, function $\Gamma: X \rightarrow 2^{\Sigma}$ (where $2^{\Sigma}$ denotes the power set of $\Sigma$ ) determines the set of active events of the states of $G$, i.e., $\Gamma(x)=\{\sigma \in \Sigma$ : $f(x, \sigma)$ is defined $\}, x_{0}$ is the initial state, and $X_{m}$ is the set of marked states. Throughout the text, $\Sigma^{*}$ denotes the Kleene closure of $\Sigma$, which is the set of all finite strings formed by events in $\Sigma$ including the empty string, denoted by $\varepsilon$. The transition function $f$ is extended to $f: X \times \Sigma^{*} \rightarrow X$ in the following manner: $f(x, \varepsilon)=x$ and $f(x, s \sigma)=f(f(x, s), \sigma)$, for all $x \in X, s \in \Sigma^{*}$ and $\sigma \in \Sigma$. The languages generated and marked by $G$ are, respectively, $L(G)=\left\{s \in \Sigma^{*}: f\left(x_{0}, s\right)\right.$ is defined $\}$ and $L_{m}(G)=\left\{s \in L(G): f\left(x_{0}, s\right) \in X_{m}\right\}$. The prefix-closure 
of a language $L$ is defined as $\bar{L}=\left\{s \in \Sigma^{*}:\left(\exists t \in \Sigma^{*}\right)[s t \in\right.$ $L]\}$, and, if $L=\bar{L}, L$ is said to be prefix-closed.

Let $\Sigma_{s}$ and $\Sigma_{l}$ be two sets of events such that $\Sigma_{s} \subseteq \Sigma_{l}$. The natural projection (Ramadge and Wonham, 1989) from $\Sigma_{l}^{*}$ to $\Sigma_{s}^{*}$ is a function that removes from strings in $\Sigma_{l}^{*}$ those events that are not in $\Sigma_{s}$, and is denoted as $P: \Sigma_{l}^{*} \rightarrow \Sigma_{s}^{*}$. The inverse projection $P^{-1}$ is the function that recovers those strings in $\Sigma_{l}^{*}$ that generate a given projection in $\Sigma_{s}^{*}$, being formally defined as $P^{-1}(t)=\left\{s \in \Sigma_{l}^{*}: P(s)=t\right\}$. The projection and the inverse projection operations are both extended to languages by applying $P$ and $P^{-1}$ to all strings in the language.

The accessible and coaccessible parts of an automaton $G$ are denoted by $A c(G)$ and $\operatorname{CoAc}(G)$, respectively, and the parallel composition between two automata $G_{1}$ and $G_{2}$ is denoted as $G_{1} \| G_{2}$. The languages generated and marked by $G_{1} \| G_{2}$ are, respectively, $L\left(G_{1} \| G_{2}\right)=P_{1}^{-1}\left[L\left(G_{1}\right)\right] \cap P_{2}^{-1}\left[L\left(G_{2}\right)\right]$ and $L_{m}\left(G_{1} \| G_{2}\right)=$ $P_{1}^{-1}\left[L_{m}\left(G_{1}\right)\right] \cap P_{2}^{-1}\left[L_{m}\left(G_{2}\right)\right]$, where $P_{i}:\left(\Sigma_{1} \cup \Sigma_{2}\right)^{*} \rightarrow \Sigma_{i}^{*}$, for $i=1,2$ (Cassandras and Lafortune, 2008, chap. 2).

The supervisor is a mapping $S: L(G) \rightarrow 2^{\Sigma}$, where, for a given string $s \in L(G), \Gamma\left[f\left(x_{0}, s\right)\right] \cap S(s)$ are the events that are enabled by $S$ at state $f\left(x_{0}, s\right)$ of $G$. The set of events $\Sigma$ is partitioned as $\Sigma=\Sigma_{c} \dot{\cup} \Sigma_{u c}$, where $\Sigma_{c}$ and $\Sigma_{u c}$ are, respectively, the sets of controllable events and uncontrollable events. In this regard, we say that a supervisor is admissible if for all $s \in L(G), \Gamma\left[f\left(x_{0}, s\right)\right] \cap$ $\Sigma_{u c} \subseteq S(s)$. The language achieved by the closed-loop system $S / G$ is, therefore, recursively characterized as: (i) $\varepsilon \in L(S / G)$, and (ii) $s \sigma \in L(S / G) \Leftrightarrow s \in L(S / G) \wedge s \sigma \in$ $L(G) \wedge \sigma \in S(s)$. The language marked by the closed-loop system is equal to the part of $L_{m}(G)$ that remains after the supervisory control action, i.e. $L_{m}(S / G)=L(S / G) \cap$ $L_{m}(G)$. A supervisor is said to be nonblocking if $L(S / G)=$ $\overline{L_{m}(S / G)}$.

A realization of a supervisor $S$ is an automaton $R=$ $\left(X_{r}, \Sigma, f_{r}, \Gamma_{r}, x_{0_{r}}, X_{r}\right)$ whose states are all marked, and is such that, for all $s \in L(G), S(s)=\Gamma_{r}\left[f_{r}\left(x_{0_{r}}, s\right)\right]$. Thus, the closed-loop behavior is characterized by $R \| G$, since $L(S / G)=L(R \| G)$ and $L_{m}(S / G)=L_{m}(R \| G)$.

A language $K \subseteq L(G)$ is controllable with respect to $L(G)$ and $\Sigma_{u c}$ if $\bar{K} \Sigma_{u c} \cap L(G) \subseteq \bar{K}$, and it is said to be closed with respect to $L_{m}(G)$ (or $L_{m}(G)$-closed) if $K=\bar{K} \cap$ $L_{m}(G)$. For a given language $K \subseteq L_{m}(G)$, there exists a nonblocking supervisor $S$ such that $L_{m}(S / G)=K$ if, and only if, $K$ is controllable and $L_{m}(G)$-closed.

The applicable language requirement $K$ is frequently given as the intersection of two (or more) elementary specification languages, i.e., $K=K_{1} \cap K_{2}$, where $K_{1}, K_{2} \subseteq$ $L_{m}(G)$. In this case, we can investigate the possibility of synthesizing nonblocking supervisors $S_{1}$ and $S_{2}$ such that $L_{m}\left(S_{1} / G\right)=K_{1}$ and $L_{m}\left(S_{2} / G\right)=K_{2}$, and thus, construct the modular control architecture where modular supervisor $S_{1} \wedge S_{2}$ is obtained by computing the conjunction of $S_{1}$ and $S_{2}$, i.e., for all $s \in L(G), S_{1} \wedge S_{2}(s)=S_{1}(s) \cap$ $S_{2}(s)$. As a consequence, $L_{m}\left(S_{1} \wedge S_{2} / G\right)=K_{1} \cap K_{2}$. Moreover, supervisor $S_{1} \wedge S_{2}$ is nonblocking if, and only if, languages $K_{1}$ and $K_{2}$ are nonconflicting, i.e., $\overline{K_{1}} \cap$ $\overline{K_{2}}=\overline{K_{1} \cap K_{2}}$.

\section{PROBLEM FORMULATION}

In this section, we propose the robot navigation problem in Industry 4.0 environments to be addressed in this paper, which extends the single-robot navigation problem considered in Gonzalez et al. (2018) to a case with multiple robots. As in Gonzalez et al. (2018), we consider that the environment is represented by an automaton model, as will be described in Subsection 3.1. In Subsection 3.2, we formally present the multi-robot navigation problem in Industry 4.0 environments.

\subsection{The Environment Automaton Model $G_{e}$}

The environment where the robot navigation is performed is represented by an automaton $G_{e}=\left(X_{e}, \Sigma_{e}, f_{e}\right.$, $\left.\Gamma_{e}, x_{0_{e}}, X_{m_{e}}\right)$, whose states in $X_{e}$ represent all possible positions and orientations (poses) a robot can assume, $\Sigma_{e}$ is the set of command events that correspond to those movements that connect the poses in $X_{e}$, the transition function $f_{e}$ and the set of active events $\Gamma_{e}$ are defined according to the environment connectivity. Finally, in order to compute a path to be followed by a mobile robot, $x_{0_{e}}$ is defined as the pose of that robot at the beginning of the task and $X_{m_{e}}$ is defined as the set of states that represent the complete execution of its task (Gonzalez et al., 2018).

Let $x_{1}, x_{n+1} \in X_{e}$. A path in automaton $G_{e}$ that takes the robot from state $x_{1}$ to state $x_{n+1}$ has the form $x_{1} \sigma_{1} x_{2} \sigma_{2} x_{3} \ldots \sigma_{n} x_{n+1}$, where, $\forall k \in\{1, \ldots, n\}, x_{k} \in X_{e}$, $\sigma_{k} \in \Sigma_{e}$ and $f_{e}\left(x_{k}, \sigma_{k}\right)=x_{k+1}$. Notice that there may exist several different paths that connect state $x_{1}$ to state $x_{n+1}$, and each of them is characterized by its corresponding string of command events $\sigma_{1} \sigma_{2} \ldots \sigma_{n} \in \Sigma_{e}^{*}$. In order to compare different paths, we define the weight function

$$
\begin{aligned}
w: \Sigma_{e} & \rightarrow R_{+}, \\
\sigma & \mapsto w(\sigma)=c,
\end{aligned}
$$

where $c$ represents the cost of executing the robot movement corresponding to command event $\sigma$. We, then, define the cost of executing a string $s=\sigma_{1} \sigma_{2} \ldots \sigma_{n} \in \Sigma_{e}^{*}$ as follows:

$$
J(s)=\sum_{i=1}^{n} w\left(\sigma_{i}\right) .
$$

Example 1. Consider the hypothetical industrial environment initially introduced in Gonzalez et al. (2018) and depicted in Figure 1, which resembles a smart factory composed by a conveyor belt (1), which is a loading and unloading terminal, shelves (5) and (6) that are used to store raw materials and parts processed either by the computer numerically controlled (CNC) milling machine (7) or the painting machines (2) and (3). The robot arm (4) is used either to transfer parts from one paint machine to the other, or to reject those painted parts that do not meet some quality standard to the orange rectangle. Notice that a mobile robot must be at pose $M_{i}$ to interact with element (i), $i=1, \ldots, 7$.

The automaton that models this environment is $G_{e}=$ $\left(X_{e}, \Sigma_{e}, \Gamma_{e}, x_{0_{e}}, X_{m_{e}}\right)$, where (i) $X_{e}=X_{i n t} \dot{\cup} X_{p s}$ with $X_{\text {int }}=\{1,2,3, \ldots, 32\}$ and $X_{p s}=\left\{M_{1}, M_{2}, M_{3}, \ldots, M_{7}\right\}$ are formed with those poses that correspond to the corridor intersections and those poses that allow the robot to pick and store parts, respectively, (ii) $\Sigma_{e}$, formed by the 


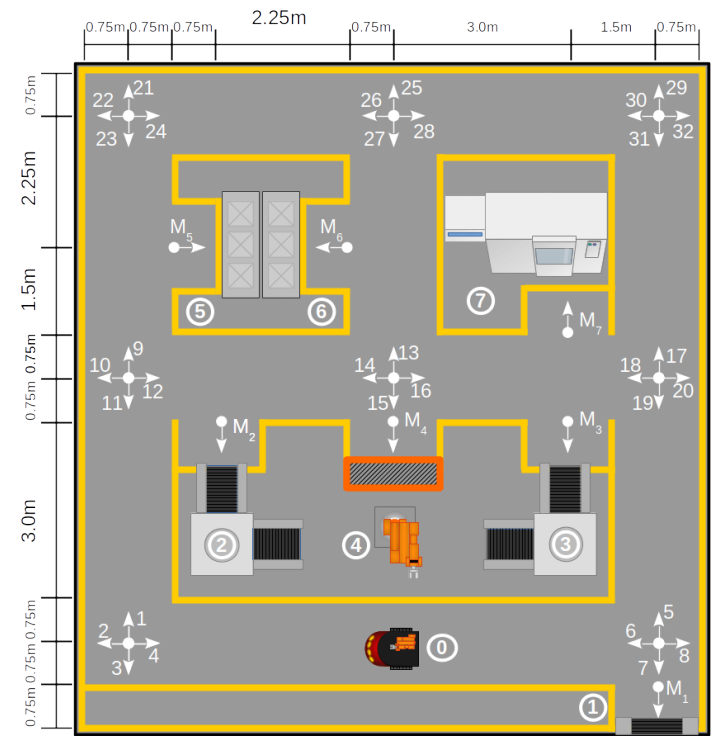

Figure 1. Map of the environment. The arrows represent the possible robot poses (states of automaton $G_{e}$ ): the tail indicates the positional coordinate and the direction corresponds to the robot orientation.

events listed in Table 1, (iii) $\Gamma_{e}: X_{e} \rightarrow 2^{\Sigma_{e}}$, presented in Table 2 for all $x \in X_{e}$; (iv) $f_{e}$, defined, for each state $x \in X_{e}$, according to the active events presented in Table 2 - for example, for $x=1$, transition function $f_{e}$ is defined for events $m 4.5, t 180, t 90$ and $t 90^{-}$, and, as it can be seen, with the help of Figure $1, f_{e}(1, m 4.5)=9$, since command event $m 4.5$ models a $4.5 \mathrm{~m}$ forward movement, $f_{e}(1, t 180)=3$, since command event $t 180$ corresponds to a $180^{\circ}$ rotational movement, and $f_{e}(1, t 90)=2$ (resp. $f_{e}\left(1, t 90^{-}\right)=4$ ) since command event $t 90$ (resp. $\left.t 90^{-}\right)$is associated with a $90^{\circ}$ counterclockwise (resp. clockwise) rotational movement, and; $(v) x_{0_{e}}$ and $X_{m_{e}}$, respectively, which are defined by the planner at each task assigned to a robot.

The values of the weight function $w$ are listed in the third column of Table 1, being defined as follows: (i) $w(m d)=$ $d+0.01$, where $d \in\{0.75,4.5,9.0\}$ is the distance to be traveled; (ii) $w(t \theta)=|\theta| / 200+0.01$, where $\theta \in\{90,180\}$ is the rotation angle, and; (iii) $w(s m k)=\sum_{i}\left(\left|\theta_{i}\right| / 200+\right.$ $0.01)+\sum_{j}\left(d_{j}+0.01\right), k=1,2, \ldots, 13$, where $\theta_{i}\left(\right.$ resp. $\left.d_{j}\right)$ are all rotational (resp. translational) movements present in $s m k$.

\subsection{Multi-robot Navigation Problem}

We address, in this paper, the problem of the navigation of multiple mobile robots in an industrial environment modeled by an automaton $G_{e}$ together with a cost function (function $J$, defined in Equation (2)). The following robot tasks are considered:

- Task 1. This task is completed when the robot reaches some state (pose) belonging to a set $X_{\text {goal }} \subseteq X_{e}$;

- Task 2. This task is completed after the robot visits all states (pose) belonging to a set $X_{\text {goal }} \subseteq X_{e}$.

Since the robot is required to visit only one state in $X_{\text {goal }}$ when it executes Task 1 , it is necessary to determine the path starting at the current state of the robot and ending
Table 1. Environment automaton events $\Sigma_{e}$.

\begin{tabular}{rll}
\hline Event & \multicolumn{1}{c}{ Description } & $w()$. \\
\hline$m 0.75$ & move robot $0.75 \mathrm{~m}$ & 0.76 \\
$m 4.5$ & move robot $4.5 \mathrm{~m}$ & 4.51 \\
$m 9.0$ & move robot $9.0 \mathrm{~m}$ & 9.01 \\
$t 90$ & turn robot $90^{\circ}$ (counterclockwise) & 0.46 \\
$t 90^{-}$ & turn robot $-90^{\circ}$ (clockwise) & 0.46 \\
$t 180$ & turn robot $180^{\circ}$ & 0.91 \\
$s m 1$ & turn robot $180^{\circ}$ and move $0.75 \mathrm{~m}$ & 1.67 \\
$s m 2$ & move $1.5 \mathrm{~m}$, turn $90^{\circ}$ and move $0.75 \mathrm{~m}$ & 2.73 \\
$s m 3$ & move $1.5 \mathrm{~m}$, turn $-90^{\circ}$ and move $0.75 \mathrm{~m}$ & 2.73 \\
$s m 4$ & move $2.25 \mathrm{~m}$, turn $90^{\circ}$ and move $0.75 \mathrm{~m}$ & 3.48 \\
$s m 5$ & move $2.25 \mathrm{~m}$, turn $-90^{\circ}$ and move $0.75 \mathrm{~m}$ & 3.48 \\
$s m 6$ & move $3.00 \mathrm{~m}$, turn $90^{\circ}$ and move $0.75 \mathrm{~m}$ & 4.23 \\
$s m 7$ & move $3.00 \mathrm{~m}$, turn $-90^{\circ}$ and move $0.75 \mathrm{~m}$ & 4.23 \\
$s m 8$ & turn $180^{\circ}$, move $0.75 \mathrm{~m}$, turn $90^{\circ}$ and move $1.5 \mathrm{~m}$ & 3.64 \\
$s m 9$ & turn $180^{\circ}$, move $0.75 \mathrm{~m}$, turn $-90^{\circ}$ and move $1.5 \mathrm{~m}$ & 3.64 \\
$s m 10$ & turn $180^{\circ}$, move $0.75 \mathrm{~m}$, turn $90^{\circ}$ and move $2.25 \mathrm{~m}$ & 4.39 \\
$s m 11$ & turn $180^{\circ}$, move $0.75 \mathrm{~m}$, turn $-90^{\circ}$ and move $2.25 \mathrm{~m}$ & 4.39 \\
$s m 12$ & turn $180^{\circ}$, move $0.75 \mathrm{~m}$, turn $90^{\circ}$ and move $3.00 \mathrm{~m}$ & 5.14 \\
$s m 13$ & turn $180^{\circ}$, move $0.75 \mathrm{~m}$, turn $-90^{\circ}$ and move $3.00 \mathrm{~m}$ & 5.14 \\
\hline
\end{tabular}

Table 2. Active events of the states of $G_{e}$.

\begin{tabular}{clcl}
\hline$X_{e}$ & \multicolumn{1}{c}{$\Gamma_{e}$} & $X_{e}$ & \multicolumn{1}{c}{$\Gamma_{e}$} \\
\hline 1 & $\left\{t 90, t 90^{-}, t 180, m 4.5\right\}$ & 21 & $\left\{t 90, t 90^{-}, t 180\right\}$ \\
2 & $\left\{t 90, t 90^{-}, t 180\right\}$ & 22 & $\left\{t 90, t 90^{-}, t 180\right\}$ \\
3 & $\left\{t 90, t 90^{-}, t 180\right\}$ & 23 & $\left\{t 90, t 90^{-}, t 180, m 4.5\right.$, \\
4 & $\left\{t 90, t 90^{-}, t 180, m 9.0\right\}$ & & $s m 4\}$ \\
5 & $\left\{t 90, t 90^{-}, t 180, m 4.5\right\}$ & 24 & $\left\{t 90, t 90^{-}, t 180, m 4.5\right\}$ \\
6 & $\left\{t 90, t 90^{-}, t 180, m 9.0\right\}$ & 25 & $\left\{t 90, t 90^{-}, t 180\right\}$ \\
7 & $\left\{t 90, t 90^{-}, t 180, m 0.75\right\}$ & 26 & $\left\{t 90, t 90^{-}, t 180, m 4.5\right\}$ \\
8 & $\left\{t 90, t 90^{-}, t 180\right\}$ & 27 & $\left\{t 90, t 90^{-}, t 180, m 4.5\right.$, \\
9 & $\left\{t 90, t 90^{-}, t 180, m 4.5, s m 5\right\}$ & & $s m 5\}$ \\
10 & $\left\{t 90, t 90^{-}, t 180\right\}$ & 28 & $\left\{t 90, t 90^{-}, t 180, m 4.5\right\}$ \\
11 & $\left\{t 90, t 90^{-}, t 180, m 4.5\right\}$ & 29 & $\left\{t 90, t 90^{-}, t 180\right\}$ \\
12 & $\left\{t 90, t 90^{-}, t 180, m 4.5, s m 3\right\}$ & 30 & $\left\{t 90, t 90^{-}, t 180, m 4.5\right\}$ \\
13 & $\left\{t 90, t 90^{-}, t 180, m 4.5, s m 4\right\}$ & 31 & $\left\{t 90, t 90^{-}, t 180, m 4.5\right\}$ \\
14 & $\left\{t 90, t 90^{-}, t 180, m 4.5, s m 6\right\}$ & 32 & $\left\{t 90, t 90^{-}, t 180\right\}$ \\
15 & $\left\{t 90, t 90^{-}, t 180, m 0.75\right\}$ & $M_{1}$ & $\{s m 1\}$ \\
16 & $\left\{t 90, t 90^{-}, t 180, m 4.5\right.$, & $M_{2}$ & $\{s m 8, s m 11\}$ \\
& $s m 6, s m 7\}$ & $M_{3}$ & $\{s m 1\}$ \\
17 & $\left\{t 90, t 90^{-}, t 180, m 4.5\right\}$ & $M_{4}$ & $\{s m 9, s m 12\}$ \\
18 & $\left\{t 90, t 90^{-}, t 180, m 4.5\right.$, & $M_{5}$ & $\{s m 10, s m 11\}$ \\
& $s m 2, s m 3\}$ & $M_{6}$ & $\{s m 10, s m 11\}$ \\
19 & $\left\{t 90, t 90^{-}, t 180, m 4.5\right\}$ & $M_{7}$ & $\{s m 8, s m 13\}$ \\
20 & $\left\{t 90, t 90^{-}, t 180\right\}$ & & \\
\hline & & & \\
\end{tabular}

at one of the states in $X_{\text {goal }}$ that minimizes the cost function $J$. On the other hand, when the robot executes Task 2, it needs to visit all states in $X_{\text {goal }}$, regardless of the ordering, by following a path that minimizes the cost function $J$. We make the following assumptions:

A1. There may exist static or mobile unpredictable obstacles in the environment, so that some transitions of the environment automaton are not allowed to fire either definitely or temporarily. If the detected obstacle is a static one, then the transition blocked by it will be blocked definitely. On the other hand, if the detected obstacle is a mobile one, possibly another robot, the transition is temporarily blocked, and thus, the robot can resume its navigation after its trajectory becomes free again.

A2. A robot does not know the position of the other robots in the environment, and it is unable to distinguish robots from other types of detectable obstacles. Thus, when a robot detects another by using its sensors, it sees that robot as an obstacle, simply.

A3. There exists a external agent, referred here to as server, that is able to communicate with all robots. 


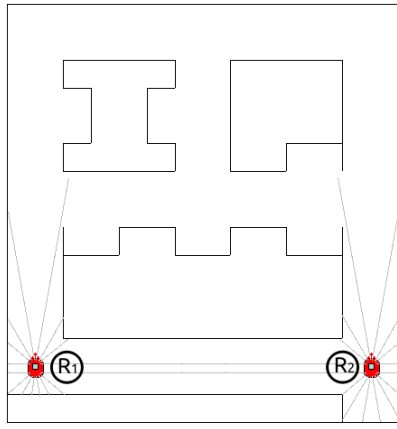

(a)

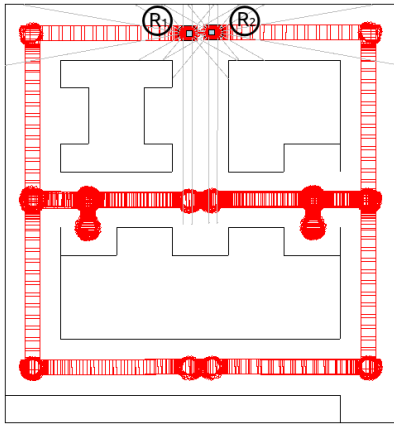

(b)
Figure 2. Problem that arises when the approach presented in Gonzalez et al. (2018) is applied to multiple robots in an environment $G_{e}$ that has corridors that allow robot movements in both directions.

At first glance, the single-robot navigation architecture proposed in Gonzalez et al. (2018) can be used to control the navigation of several robots in the same environment. However, this approach may fail, as illustrated by the following example.

Example 2. Consider the same navigation environment addressed in Example 1 and shown in Figure 1. Assume that two robots $R_{1}$ and $R_{2}$ are operating in this environment, and their initial poses are states 1 and 5 , respectively, as shown in Figure 2(a).

Consider that robot $R_{1}$ receives a task to go to state 5 and robot $R_{2}$ receives a task that demands it to go to state 1. According to Gonzalez et al. (2018), the planners of both robots calculate the shortest paths to their goals, and thus, both computed trajectories pass through the bottom horizontal corridor. As a consequence, the robots will meet, detect each other and stop their movements. As both robots wait for each other to move away, they both consider that the other robot is a static obstacle. Then, robots $R_{1}$ and $R_{2}$ return to states 4 and 6 , respectively, and recalculate the shortest path to their goals by discarding the bottom horizontal corridor as a viable trajectory. This will repeat itself in the middle and top corridors, resulting in a feasible task being deemed as impossible. The final result can be seen in Figure 2(b).

\section{A DES-BASED MULTI-ROBOT NAVIGATION ARCHITECTURE}

In this section, we propose a modified version of the navigation architecture proposed in Gonzalez et al. (2018) to tackle multi-robot navigation. Such a new approach adds new features to the automaton models of the robots, in order to address possible problems that arises when two robots meet facing each other.

Notice that, a possible approach to circumvent problems that occur when two robots meet facing each other is prevent such meetings. In this regard, a possible solution would be to distribute the robot tasks in such a way that two, or more, robots do not move in the same area at the same time. This solution would require either a collective trajectory planning or a collective scheduling method. However, such a solution may be conservative in the sense that it increases the computational complexity

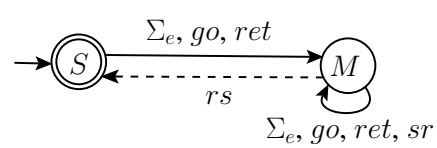

Figure 3. The robot movement module $G_{r_{m}, i}$. Dashed lines represent transitions labeled with uncontrollable events.

Table 3. Robot movement module events.

\begin{tabular}{clc}
\hline Event & \multicolumn{1}{c}{ Description } & Controllable \\
\hline$\Sigma_{e}$ & set of environment automaton events & $\checkmark$ \\
$s r$ & stop the robot & $\checkmark$ \\
$r e t$ & return to the last visited state & $\checkmark$ \\
$g o$ & complete the last movement & $\checkmark$ \\
$r s$ & robot stopped & $\times$ \\
\hline
\end{tabular}

due to the centralized planning/scheduling, and, as larger the area reserved for a given robot, as longer may be the paths computed for the other robots.

An alternative approach is to create a method of deciding, whenever two robot meet, which robot must recalculate its trajectory and which one should wait until the path is free to resume its previously calculated path. This can be done either by a local decision made between the robots or by another agent. Since it is already assumed that there are external agents that assign tasks to the robots, we will assume that there is a server that receives obstacle detection signals from all robots together with the information of their current positions and their distances to their task goals. Thus, if two robots are in poses that imply they have met head-on, the server will identify that the robots have met each other and will ask for the robot that is further away from its goal to recalculate its path, while the other robot will be allowed to continue following the previously planned trajectory. This solution is easier in terms of adapting the architecture developed in Gonzalez et al. (2018), since we only need to change the robot automaton model, $G_{r}$, and a specification automaton, $H_{\text {spec,3 }}$, of the navigation problem.

\subsection{The Robot Model $G_{r, i}$}

In order to construct a discrete event model for the $i$-th robot, the features that are important for the correct planning and execution of the navigation task are separately modeled by using automata. In this paper, we propose a robot automaton model, denoted by $G_{r, i}$, which is obtained by performing the following parallel composition:

$$
G_{r, i}=G_{r_{m}, i}\left\|G_{r_{s}, i}\right\| G_{r_{t m}, i},
$$

where $G_{r_{m}, i}, G_{r_{s}, i}$ and $G_{r_{t m}, i}$ model the robot movement, sensing and task manager modules of the $i$-th robot, respectively.

- Robot movement module. Automaton $G_{r_{m}, i}$, depicted in Figure 3, models the robot movement resources. The events of $G_{r_{m}, i}$ are listed in Table 3. In order to navigate in a given industrial environment, the robot must be able to execute the events in $\Sigma_{e}$. In addition, it also requires other command events to deal with unpredictable obstacles, such as, event $s r$, used to stop the robot in order to prevent collisions, and events ret and go, used to return the robot to the last visited state and to complete the movement interrupted by the obstacle, respectively. Automaton 


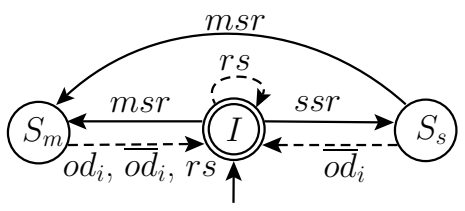

Figure 4. The robot sensing module $G_{r_{s}, i}$. Dashed lines represent transitions labeled with uncontrollable events.

Table 4. Robot sensing module events.

\begin{tabular}{clc}
\hline Event & \multicolumn{1}{c}{ Description } & Controllable \\
\hline$r s$ & $\begin{array}{l}\text { robot stopped } \\
\text { obstacle sensor information request } \\
\text { while the robot is moving }\end{array}$ & $\checkmark$ \\
$s s r$ & $\begin{array}{l}\text { obstacle sensor information request } \\
\text { when the robot has stopped }\end{array}$ & $\checkmark$ \\
$o d_{i}$ & $\begin{array}{l}\text { The } i \text {-th robot detects an obstacle and } \\
\text { sends a message to the server with the }\end{array}$ & $\times$ \\
& $\begin{array}{l}\text { its pose and its distance from the } \\
\text { task objective }\end{array}$ \\
$\overline{o d}_{i}$ & $\begin{array}{l}\text { message sent by the } i \text {-th robot to the } \\
\text { server, to inform it does not } \\
\text { detect obstacles }\end{array}$ \\
\hline
\end{tabular}

$G_{r / m}, i$ has also the uncontrollable event $r s$, which is due to sensor readings, and represents the transition from state $M$, where the robot is moving, to state $S$, where the robot is stopped.

Its worth noticing that the events that belong to $\Sigma$ are controllable, and can be cancelled either by the supervisor or by the occurrence of a new movement command.

- Robot sensing module. Automaton $G_{r_{s}, i}$, depicted in Figure 4, models the robot sensing resources. It is assumed that the robot has wheel encoders and at least one sensing system that is able to detect obstacles, such as sonars, laser rangefinders or vision-based systems. The wheel encoders are used to determine when event $r s$ occurs, that is, when the robot stops after finishing the last movement command. The obstacle detection sensors are used to monitor the presence of unknown obstacles that block the robot path, by means of command events $m s r$ and $s s r$. Command event $m s r$ is used to request the execution of a sensing routine when the robot is moving, which, then, returns event $o d_{i}, \overline{o d}_{i}$ or $r s$ (active event set of state $S_{m}$ ). Event $o d_{i}\left(\right.$ resp. $\overline{o d}_{i}$ ) indicates that an obstacle is detected (resp. no obstacle detected), and event $r s$ indicates that the robot stopped and, consequently, the sensor reading is no longer necessary. Command event $s s r$ is used, when the robot is stopped, to start a sensing routine that keeps $G_{r_{s}, i}$ in state $S_{s}$ until a $m s r$ event or no obstacle detection, represented by event $\overline{o d}_{i}$, occurs. The complete list of events of $G_{r_{s}, i}$ is presented in Table 4 . Notice that events $m s r$ and $s s r$ are controllable, whereas events $r s, o d_{i}$ and $\overline{o d}_{i}$ are uncontrollable.

- Robot task manager module. Automaton $G_{r_{t m}, i}$, depicted in Figure 5, models the robot resources associated with the management of the robot task. It has four states which represent the current robot status regarding task management, as follows: (i) robot idle $(I)$, (ii) planning the robot trajectory $(P)$, (iii) executing the task $(W)$, and $(i v)$ waiting for the removal of an obstacle $(B)$. The events of $G_{r_{t m}, i}$ are listed in Table 5 . Event $n t_{i}$ is issued

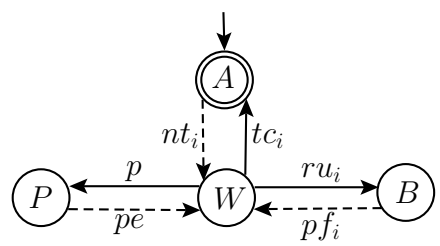

Figure 5. The robot task manager module $G_{r_{t m}, i}$. Dashed lines represent transitions labeled with uncontrollable events.

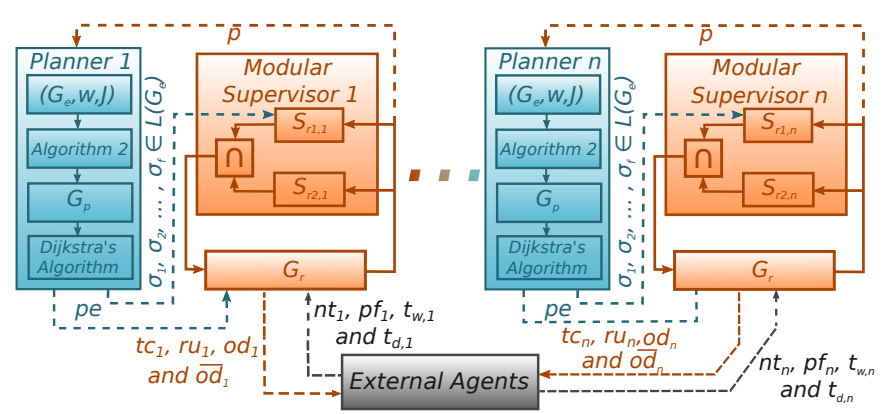

Figure 6. The proposed navigation architecture.

by an external agent requesting the execution of a new task by robot $i$. Command event $p$ is used to start the path planning procedure. After that, the planner starts to execute the path planning and when it is completed, the planner sends to the robot a signal, which corresponds to the occurrence of event pe. Command event $r u_{i}$ (request for unblocking the path) is a robot request to an external agent to remove the obstacle between the current robot position and the last visited state. When the external agent removes the obstacle, it issues event $p f_{i}$, meaning that the path is free. Thus, events $t c_{i}, p$ and $r u_{i}$ are controllable, and events $n t_{i}$, pe and $p f_{i}$ are uncontrollable.

Table 5. Robot task manager module events.

\begin{tabular}{clc}
\hline Event & \multicolumn{1}{c}{ Description } & Controllable \\
\hline$n t_{i}$ & the $i$-th robot received a new task & $\times$ \\
$t c_{i}$ & the $i$-th robot reports task completion & $\checkmark$ \\
$p$ & execute the planning & $\checkmark$ \\
$p e$ & planning concluded & $\times$ \\
$r u_{i}$ & the $i$-th requests for unblocking the last path & $\checkmark$ \\
$p f_{i}$ & the last path of the $i$-th robot is free & $\times$ \\
\hline
\end{tabular}

\subsection{The Navigation Process}

The multi-robot navigation architecture proposed in this paper consists of several robots, where each one of them is controlled by a modular supervisory controller, as shown in Figure 6. An advantage of this structure is that it allows a decentralized implementation, where the modular supervisor is embedded in the mobile robots, whereas the planner may run in external agents, which suits very well to the design problem addressed here, since it makes easier to adapt the proposed navigation architecture to other environments. In addition, the navigation architecture has also a specific type of external agent, called server, that is able to communicate with all robots (Assumption A3). The server is responsible for solving conflicts between the robots, which are configured to send to it their obstacle detection signals, together with the information of their current positions and their distances to their task goals. 
The navigation process starts when the $i$-th robot is available and an external agent assigns a new task to it. This assignment is modeled by the occurrence of event $n t_{i}$ of automaton $G_{r_{m t, i}}$ of Figure 5. Then, robot $G_{r, i}$ generates event $p$ (execute planning), which carries the following information: (i) the robot current state; (ii) the last task assigned to the robot and its respective set of target states $X_{\text {goal }}$, and; (iii) set $T_{b, i}$, which is formed with those transitions identified as blocked, being initialized as an empty set and modified by the robot when event $o d_{i}$ occurs in $G_{r_{s}, i}$, i.e., the blocked transition of $G_{e}$ is added to $T_{b, i}$ when the $i$-th robot detects a permanent obstacle. The blocked transition is determined from the last state of $G_{e}$ visited by the robot and the command event in $\Sigma_{e}$ whose execution was interrupted by the obstacle detection.

According to the diagram depicted in Figure 6, when the planner receives event $p$, it runs a computer application to determine the path to be followed by the robot. Notice that the planner is composed by environment automaton $G_{e}$, and weight and cost function, $w$ and $J$, defined in accordance with Equations (1) and (2), respectively.

After the planning is finished, the planner sends event $p e$ to automaton $G_{r, i}$ to inform that the planning has been concluded. The planner also sends the string of command events that corresponds to the computed path to be used to design supervisor $S_{r_{1, i}}$; thus, a new supervisor $S_{r_{1, i}}$ is computed after each new path planning completion.

The modular supervisory control $S_{r_{1, i}} \wedge S_{r_{2, i}}$ ensures the correct navigation of the $i$-th robot in the presence of permanent or intermittent unpredictable obstacles, and of other robots. $S_{r_{1, i}}$ acts so as to enforce the robot to follow the path computed by the planner, whereas $S_{r_{2, i}}$ ensures that design specifications $S P_{1}-S P_{9}$ (to be presented in Subsection 4.4) are achieved.

\subsection{Path Planning and Conflict Solving Procedures}

The path planning procedure is the same proposed in Gonzalez et al. (2018), but it is worth remarking that each robot will calculate its trajectory regardless of the presence of other robots, since the position of the other robots are unknown to it.

Although each path planning process is done separately, whenever two robots meet facing each other, the server decides which robot will have to return to the previous state and recalculate its trajectory. The criteria used to choose which robot recalculates its trajectory is a project parameter. The criterion adopted in this article is the closeness of involved robots to their goals. Namely, the robot farthest from its object will recalculate its trajectory, by considering the other robot as a static obstacle, while the other robot waits its path to be free.

Consider that two robots $R_{1}$ and $R_{2}$ operate in the same environment, and meet facing each other. Suppose that $R_{1}$ is the robot that is closer to its task goal. When both robots detect each other, they send to the server a signal warning they have encountered unknown obstacles, together with the information regarding their positions and their distances to their respective goals. Then, the server infers that a encounter between $R_{1}$ and $R_{2}$ has occurred. Since robot $R_{1}$ is closer to its goal, $R_{2}$ will receive

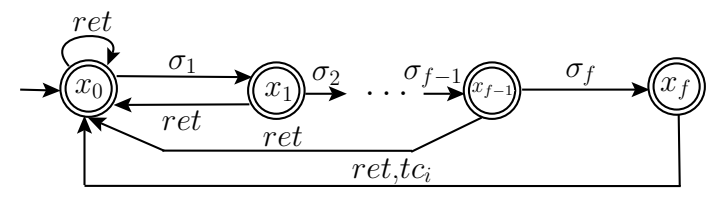

Figure 7. Automaton $H_{\text {spec,1 }}$ used to synthesize supervisor $S_{r_{1, i}}$.

a signal telling it to recalculate its trajectory, while $R_{1}$ waits until $R_{2}$ leaves its path. The server tell $R_{1}$ when its path is free, based on the information of the last state $x_{\text {last }}$ of $G_{e}$ visited by it, and the current state of $R_{2}$. Namely, we assume that the server has a list $\ell_{\text {server }}(x)$, for each state $x \in X_{e}$, that stores those states where the presence of $R_{2}$ disturb the trajectory of $R_{1}$. Thus, the server only allows the movement of $R_{1}$ when $x_{2} \notin \ell_{\text {server }}\left(x_{1}\right)$, where $x_{1}$ and $x_{2}$ denote the current states of $R_{1}$ and $R_{2}$, respectively. The total cost of such a verification method is $O\left(\left\|X_{e}^{2}\right\|\right)$.

\subsection{Design of Modular Supervisor $S_{r_{1, i}} \wedge S_{r_{2, i}}$}

In order to design supervisors $S_{r_{1, i}}$ and $S_{r_{2, i}}$, we initially construct simple automata that capture the essence of the specifications we want to ensure by using these supervisors. We, then, combine these automata with $G_{r, i}$ using the parallel composition to obtain the system desired behavior.

Let $s=\sigma_{1} \ldots \sigma_{i} \sigma_{i+1} \ldots \sigma_{f} \in L\left(G_{e}\right)$ be the last string of command events computed by the planner of the $i$-th robot, the design of $S_{r_{1, i}}$ is performed from string $s$, in the same way as proposed in Gonzalez et al. (2018). Namely, we initially define the specification automaton $H_{\text {spec }, 1, i}=$ $\left(X_{1, i}, \Sigma_{1, i}, f_{1, i}, \Gamma_{1, i}, x_{0_{1, i}}, X_{1, i}\right)$ depicted in Figure 7 . In the sequel, we compute automaton $H_{1, i}=G_{r, i} \| H_{s p e c, 1, i}$ that marks the applicable language requirement $K_{1, i}$. It is worth remarking that the set of events of automaton $G_{r, i}$ is $\Sigma_{r}=\Sigma_{e} \cup\{s r, r e t, g o, r s, m s r, s s r, o d, \overline{o d}, t, n t, t c, p$, $p e, r u, p f\}$. Thus, if $P_{1}: \Sigma_{r}^{*} \rightarrow \Sigma_{1}^{*}$, we can state that:

$$
K_{1, i}=P_{1}^{-1}\left[L_{m}\left(H_{\text {spec }, 1, i}\right)\right] \cap L_{m}\left(G_{r, i}\right) .
$$

It is not difficult to conclude that $K_{1, i}$ is controllable. In addition, because all states of $H_{s p e c, 1, i}$ are marked, $K_{1, i}$ is, by construction, $L_{m}\left(G_{r, i}\right)$-closed. Finally, an automaton realization of a nonblocking supervisor $S_{r_{1, i}}$ such that $L_{m}\left(S_{r_{1, i}} / G_{r, i}\right)=K_{1, i}$ is obtained from $H_{s p e c, 1}$ by adding self-loops labeled by the events in $\Sigma_{r} \backslash \Sigma_{1, i}$ to all of its states.

Let us now consider the synthesis of supervisor $S_{r_{2, i}}$, which deals, among other requirements, with static and mobile (possibly other robots) obstacles. In order to achieve the desired behavior, the following specifications are enforced:

- $S P_{1}$ The robot can perform the command events in $\Sigma_{e} \cup\{$ ret, go $\}$ only after it receives a new task and the trajectory has already been planned.

- $\mathrm{SP}_{2}$ After the execution of command event ret, which, according to specification $S P_{6}$, will only be executed after the detection of a permanent obstacle (possibly another robot), the robot cannot perform movement commands in $\Sigma_{e} \cup\{g o\}$ before a new trajectory is computed by the planner.

- $S P_{3}$ In order to prevent the wrong functioning of low level controllers that execute the robot movements, a 
command event in $\Sigma_{e} \cup\{$ ret, go $\}$ cannot be sent before the execution of the previous movement has been either completed or aborted by command $s r$ (stop the robot).

- $\mathrm{SP}_{4}$ In order to prevent collisions, the robot continuously checks the existence of obstacles in the trajectory, and, when an obstacle is detected, it must stop.

- $S P_{5}$ After the robot stops due to an obstacle detection, it must notify the server it detected and obstacle. The server will, then, distinguish if the obstacle is a robot or not. If the obstacle is not another robot, the robot must wait a given time to check if it moves away. If the obstacle goes away before the waiting time is over, it must send an $\overline{o d}_{i}$ signal to the server, which will, in return, tell the robot it can resume its navigation.

- $S P_{6}$ When the server infers the robot has detected a permanent obstacle, the robot must return to the last visited state in $G_{e}$, which corresponds to the last pose visited in the environment, by using command event ret.

- $S P_{7}$ When the robot detects a permanent obstacle while it executes the movements associated with command event ret, it must request an external agent to remove this obstacle by means of event $r u$.

- $S P_{8}$ When the $i$-th robot detects another robot, and the $i$-th robot is farther from its task goal, then, it must return to the last visited state in $G_{e}$, which corresponds to the last pose visited in the environment, by using command event ret.

- $S P_{9}$ When the $i$-th robot detects another robot, but the $i$-th robot is closer to its task goal, then, it must wait until the detected robot moves away. After the detected robot has gone out of the sensor's range, the $i$-th robot must inform this to the server, which will send a $t_{d}$ signal informing when the $i$-th robot will resume its navigation.

Specifications $S P_{1}-S P_{4}$ and $S P_{7}$ have been also imposed in Gonzalez et al. (2018). On the other hand, the new specifications $S P_{5}, S P_{6}, S P_{8}$ and $S P_{9}$ establishes the interaction between the mobile robots and the server. We will now construct the specification automata that capture the essence of specifications $S P_{i}, i=1, \ldots, 9$. To this end, we first construct automaton $H_{\text {spec }, 2, i}$ depicted in Figure 8, whose set of events is $\Sigma_{2}=\Sigma_{e} \cup\left\{n t_{i}, p, p e, t c_{i}\right.$,ret, go $\}$ and accounts for specifications $S P_{1}$ and $S P_{2}$. In the sequel, we compute automaton $H_{\text {spec }, 3, i}$, depicted in Figure 9, that accounts for specifications $S P_{3}-S P_{9}$. It is worth remarking that automaton $H_{\text {spec }, 3, i}$ differs from automaton $H_{\text {spec }, 3}$ computed in Gonzalez et al. (2018), since the former accounts for the new specifications $S P_{5}, S P_{6}, S P_{8}$ and $S P_{9}$. The event set of $H_{\text {spec }, 3, i}$ is $\Sigma_{3}=\Sigma_{r} \backslash\{n t, p e\}$, and its states correspond to the following situations: (i) state 0 represents the case when the robot has stopped without detecting obstacles; (ii) states 1 and 2 correspond to the case when the robot is executing the movements associated with the command events in $\Sigma_{e}$; and (iii) states 3 to 16 are associated with the procedure to handle obstacles.

Automaton $H_{2, i}$ that marks the applicable language requirement $K_{2, i}$ is computed by performing the parallel composition $H_{2}=G_{r, i}\left\|H_{\text {spec }, 2, i}\right\| H_{\text {spec }, 3, i}$. Thus, by defining projections $P_{2}: \Sigma_{r}^{*} \rightarrow \Sigma_{2}^{*}$ and $P_{3}: \Sigma_{r}^{*} \rightarrow \Sigma_{3}^{*}$, then,

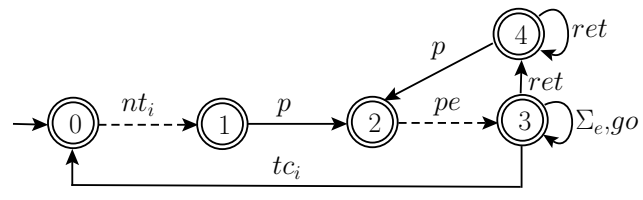

Figure 8. Automaton $H_{s p e c, 2, i}$ used to synthesize supervisor $S_{r_{2, i}}$. Dashed lines represent transitions labeled with uncontrollable events.

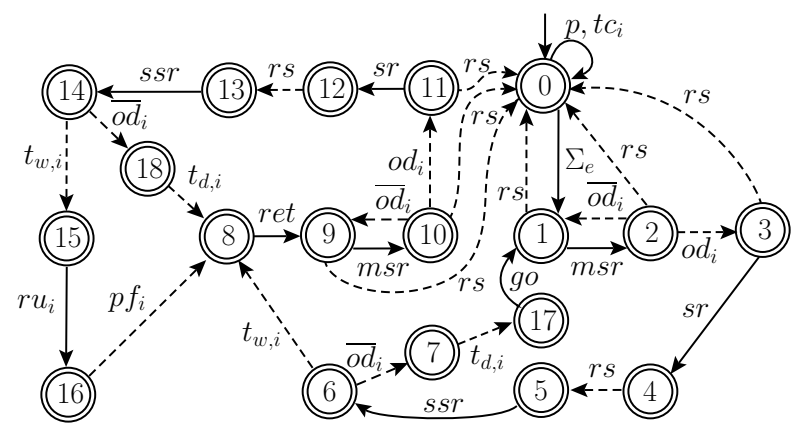

Figure 9. Automaton $H_{\text {spec,3,i }}$ used to synthesize supervisor $S_{r_{2, i}}$ for the navigation of multiple robots. Dashed lines represent transitions labeled with uncontrollable events.

$K_{2, i}=L_{m}\left(G_{r, i}\right) \cap P_{2}^{-1}\left[L_{m}\left(H_{s p e c, 2, i}\right)\right] \cap P_{3}^{-1}\left[L_{m}\left(H_{s p e c, 3, i}\right)\right]$.

It can be check that $K_{2, i}$ is controllable and $L_{m}\left(G_{r, i}\right)$ closed. In addition, An automaton realization of a nonblocking supervisor $S_{r_{2, i}}$ such that $L_{m}\left(S_{r_{2, i}} / G_{r, i}\right)=$ $K_{2, i}$ is obtained by calculating the parallel composition $H_{\text {spec }, 2, i} \| H_{\text {spec }, 3, i}$. Notice that the set of events of $H_{\text {spec }, 2, i} \| H_{\text {spec }, 3, i}$ is equal to $\Sigma_{r}$, and, thus, we do not need to add self-loops to it in order to obtain a realization of supervisor $S_{r_{2, i}}$.

According to modular supervisory control theory presented in Section 2, the modular supervisory architecture constructed by the conjunction of $S_{r_{1, i}}$ and $S_{r_{2, i}}$ is such that $L_{m}\left(S_{r_{1, i}} \wedge S_{r_{2, i}} / G_{r, i}\right)=K_{1, i} \cap K_{2, i}$, where $K_{1, i}$ and $K_{2, i}$ are defined in Equations (4) and (5), respectively. In addition, it can be verified that the admissible languages $K_{1, i}$ and $K_{2, i}$ are nonconflicting, which ensures that the conjunctive modular supervisor $S_{r_{1, i}} \wedge S_{r_{2, i}}$ is nonblocking, i.e., $L\left(S_{r_{1, i}} \wedge S_{r_{2, i}} / G\right)=\overline{K_{1, i} \cap K_{2, i}}$.

Example 3. Consider, again, the problem addressed in Example 2, where two robots $R_{1}$ and $R_{2}$ are operating in the hypothetical industry 4.0 environment shown in Figure 1, being controlled by the navigation architecture proposed in Section 4. The initial pose of robot $R_{1}$ (resp. robot $R_{2}$ ) is state 1 (rep. state 5 ), as shown in Figure 2(a), and its task is to go to state 5 (resp. state 1 ).

Initially, the planners of both robots calculate the shortest paths to their goals, and thus, both computed trajectories pass through the bottom horizontal corridor. As illustrated in Figure 10, when the robots are facing each other in that corridor, the server, then, determines that $R_{1}$ and $R_{2}$ have encountered each other, and, since $R_{1}$ is closer to its goal, it will wait for a timeout signal from the server for completing its task. Meanwhile, robot $R_{2}$ will, turn $180^{\circ}$, move to state 8 , turn $180^{\circ}$ again, and recalculate the shortest path from its current pose, state 6 , to state 


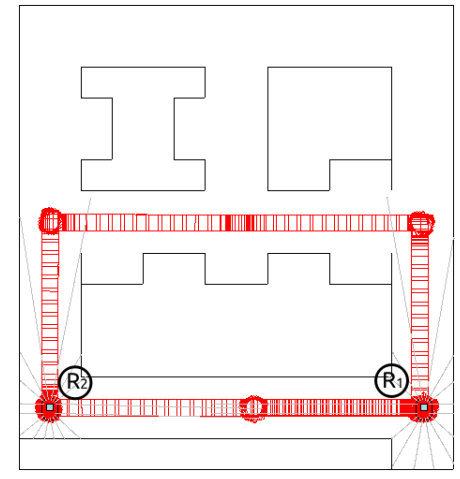

Figure 10. Result obtained with the proposed approach.

1 , by considering that the transition from state 6 to state 2 (which accounts for the bottom horizontal corridor) is blocked. Thus, robot $R_{2}$ reaches its goal (State 1) passing through the middle horizontal corridor instead of the bottom one, whereas robot $R_{1}$ reaches its goal (State 5 ) passing through the bottom horizontal corridor.

\section{CONCLUDING REMARKS}

In this paper, we extend the single-robot DES-based navigation architecture previously proposed in Gonzalez et al. (2018), to deal with the navigation of multiple mobile robots. We consider several robots navigating independently of each other, in an Industry 4.0 environment where there may exist static and dynamic obstacles, that are a priori unknown by the robots. Simulated experiments illustrate, in a hypothetical Industry 4.0 environment, that the single robot navigation architecture presented in Gonzalez et al. (2018) was extended by incurring little extra computational effort, since each robot computes its path independently. The modular design proposed in this paper provides an approach that scales better than that presented in Tatsumoto et al. (2018), since the computational cost of their approach grows exponentially with the number of robots. On the other hand, Hill and Lafortune (2017) did not provide a computational complexity analisys, however, its multi-level hierarchical architecture requires complex procedures to obtain abstractions of the supervisors. Moreover, none of the approaches proposed in Dulce-Galindo et al. (2019); Hill and Lafortune (2017); Tatsumoto et al. (2018) and Basile et al. (2019) is able to deal with unknown obstacles.

It is worth remarking that, although the achieved overall computational cost does not increase significantly with the number of robots, our approach can cause unwanted robot encounters, that demands additional computation to be solved. This drawback shall be tackled in future works. Another possible future work is include the distribution of the tasks for the robots (Dulce-Galindo et al., 2019; Basile et al., 2019).

\section{REFERENCES}

Basile, F., Chiacchio, P., and Marino, E.D. (2019). An auction-based approach to control automated warehouses using smart vehicles. Control Engineering Practice, 90, $285-300$.
Cassandras, C.G. and Lafortune, S. (2008). Introduction to Discrete Event Systems. Springer, New York, 2nd edition.

Da Xu, L., He, W., and Li, S. (2014). Internet of things in industries: A survey. IEEE Transactions on Industrial Informatics, 10(4), 2233-2243.

Dulce-Galindo, J.A., Santos, M.A., Raffo, G.V., and Pena, P.N. (2019). Autonomous navigation of multiple robots using supervisory control theory. In 18th European Control Conference (ECC), 3198-3203. Naples, Italy.

Fabre, E. and Jezequel, L. (2009). Distributed optimal planning: an approach by weighted automata calculus. In Proc. of the 48th IEEE Conference on Decision and Control held jointly with the 28th Chinese Control Conference (CDC/CCC), Shanghai, China, 211-216.

Gilchrist, A. (2016). Industry 4.0: the industrial internet of things. Apress, Berkeley, 1st edition.

Gonzalez, A.G.C., Alves, M.V.S., Viana, G.S., Carvalho, L.K., and Basilio, J.C. (2018). Supervisory controlbased navigation architecture: A new framework for autonomous robots in industry 4.0 environments. IEEE Transactions on Industrial Informatics, 14(4), 17321743.

Hill, R. and Lafortune, S. (2017). Scaling the formal synthesis of supervisory control software for multiple robot systems. In Proc. of the American Control Conference $(A C C)$, Seattle, WA, USA, 3840-3847.

Iqbal, J., Khan, S., Zafar, N., and Ahmad, F. (2012). Modeling supervisory control of autonomous mobile robots using graph theory, automata and z notation. Journal of American Science, 8(12), 799-804.

Jirkovsky, V., Obitko, M., and Marik, V. (2017). Understanding data heterogeneity in the context of cyberphysical systems integration. IEEE Transactions on Industrial Informatics, 13(2), 660-667.

Kehoe, B., Patil, S., Abbeel, P., and Goldberg, K. (2015). A survey of research on cloud robotics and automation. IEEE Transactions on Automation Science and Engineering, 12(2), 398-409.

Kloetzer, M. and Mahulea, C. (2016). Multi-robot path planning for syntactically co-safe LTL specifications. In Proc. of the International Workshop on Discrete Event Systems (WODES), Xi'an, China, 452-458.

Košecká, J. and Bajcsy, R. (1994). Discrete event systems for autonomous mobile agents. Robotics and Autonomous Systems, 12, 187-198.

Ramadge, P.J.G. and Wonham, W.M. (1989). The control of discrete event systems. Proceedings of the IEEE, $77(1), 81-98$.

Roszkowska, E. (2002). Supervisory control for multiple mobile robots in 2D space. In Proc. of the International Workshop on Robot Motion and Control, (RoMoCo), Bukowy Dworek, Poland, 187-192.

Tatsumoto, Y., Shiraishi, M., Cai, K., and Lin, Z. (2018). Application of online supervisory control of discreteevent systems to multi-robot warehouse automation. Control Engineering Practice, 81, 97 - 104.

Wang, S., Wan, J., Zhang, D., Li, D., and Zhang, C. (2016). Towards smart factory for industry 4.0: a self-organized multi-agent system with big data based feedback and coordination. Computer Networks, 101, 158-168. 